\title{
Failure mechanism of PMI foam core sandwich beam in bending
}

\author{
Bo Wang ${ }^{1, *}$, Yunfeng $\mathrm{Shi}^{1}$, Caihua Zhou ${ }^{1}$, and Tong $\mathrm{Li}^{2}$ \\ 1 Faculty of Vehicle Engineering and Mechanics, Dalian University of Technology, Dalian 116024, China \\ 2 School of Chemistry, Physics and Mechanical Engineering, Queensland University of Technology, 2 George St., Brisbane, \\ QLD 4001, Australia
}

Received 11 December 2015 / Accepted 18 December 2015

\begin{abstract}
Polymethacrylimide (PMI) foams have been widely applied in aerospace engineering as the core material of sandwich structures. This paper proposes a modified model to predict the constitutive relation of PMI foams and compares it to existing testing data. The study is then applied to the investigation of the failure mechanism of PMI foam core sandwich beams in bending. Corresponding bending tests were carried out where a complex failure process was observed through a high-speed camera. Numerical model of the foregoing sandwich beam is developed, in which the maximum principal stress criteria is used to predict damage propagation in PMI foam core. Both results from tests and numerical simulation validate the reliability of the theoretical prediction of the failure of PMI foam core sandwich beam using the proposed modified model of PMI foams. This study provides a theoretic tool for the design of sandwich structures with PMI foam core.
\end{abstract}

Key words: Foams, Damage mechanics, Finite element analysis, Process monitoring.

\section{Introduction}

The ROHACELL WF series polymethacrylimide (PMI) foam has been introduced as the material of structural sandwich core for aerospace structures since 1971 and today this material is listed in more than 170 aerospace specifications worldwide [1]. Besides its high specific strength and specific stiffness, the inotropic and closed-cell character of PMI foams provides a dramatic improvement over other foam materials like aluminum foams [1]. However, despite the outstanding properties of PMI foams, difficulties exist in the full understanding the failure mechanism of sandwich structures with PMI foam cores, especially under complex loading conditions. Considerable studies have been carried out on the failure analysis of PMI foams. Li and Mines have studied the compressive process of PMI foam under uniaxial compression [2] and the crush behavior under a series of tests [3]. Chen et al. have investigated the influence of foam structure on the macroscopic properties of PMI foam [4]. The fatigue behaviors of the PMI foam in tension, compression and shear were investigated by Burman and Zenkert [5-7] as well as the impact responses [8-11]. In addition, the basic mechanical properties of sandwich structures with PMI foam core were also characterized $[5,7,12-22]$, where the sandwich beams or panels with PMI foam core have been tested in standard or specialized tests such

*e-mail: wangbo@dlut.edu.cn as shearing $[7,14,20]$, three point bending $[16,18,20]$, four point bending [5, 12, 14], edgewise compression [20, 22] and indentation $[17,21]$. According to these studies, the foam's density has significant influence on the failure modes of the sandwich structures [17]. The most common failure mode of the PMI foam core sandwich structures is core shearing $[7,12,13,16,22]$, followed by local indentation collapse [16-18, 21] and face yielding [7, 22]. However, among these studies, there is a lack of systematical research on the PMI foam core sandwich structures' failure mechanism in terms of the mechanical properties of foam, such as the foam density. Limited study was conducted on the modeling of damage propagation in PMI foam cores. For the above-mentioned reasons, this paper aims at investigating the failure mechanism of sandwich beams with PMI foam core in three point bending test by applying the cellular solids theory [23]. Corresponding numerical simulations and tests were carried out to validate the theoretical prediction.

In this paper, the constitutive relation of the PMI foam is studied based on the microscopic mechanics of cellular materials introduced by Gibson and Ashby [23]. The conclusion of constitutive relationship is then applied to the theoretical investigation of failure mechanism of the sandwich beams with PMI foam core in three point bending. Moreover, three point bending tests of the sandwich beams with PMI foam core and carbon fiber skin sheets were carried out with a high speed camera to capture the beams' failure process. 


\begin{tabular}{|c|c|}
\hline \multicolumn{2}{|c|}{ Nomenclature } \\
\hline$A_{1}, A_{2}, B_{1}, B_{2}$ & Modified geometric coefficients of foam cell \\
\hline$C_{1}, C_{1}^{\prime}, C_{5}, C_{5}^{\prime \prime}$ & Geometric coefficients of foam cell \\
\hline$E^{*}$ & Young's modulus of foam material \\
\hline$E_{f}$ & Young's modulus of fiber's principal direction \\
\hline$E_{f 3}$ & Young's modulus of fiber's out-plane direction \\
\hline$E_{s}$ & Young's modulus of foam's cell-wall material \\
\hline$(E I)_{e q}$ & Equivalent flexural rigidity of sandwich beam \\
\hline$M$ & Moment applied on the sandwich beam \\
\hline$p_{0}$ & Initial fluid pressure of foam cell \\
\hline$p_{a t}$ & Atmospheric pressure \\
\hline$P$ & $\begin{array}{l}\text { Concentrated force applied on the sandwich } \\
\text { beam }\end{array}$ \\
\hline$Q$ & Shear applied on the sandwich beam \\
\hline$\varepsilon_{D}$ & $\begin{array}{l}\text { Critical strain at which the densification of } \\
\text { foam starts }\end{array}$ \\
\hline$v^{*}$ & Poisson's ratio of foam material \\
\hline$\rho^{*}$ & Density of foam material \\
\hline$\rho_{s}$ & Density of foam's cell-wall material \\
\hline$\sigma_{c}$ & Normal stresses in the core of sandwich beam \\
\hline$\sigma_{f}$ & Normal stresses in the faces of sandwich beam \\
\hline$\sigma_{p l}^{*}$ & $\begin{array}{l}\text { Compressive crushing strength of foam } \\
\text { material }\end{array}$ \\
\hline$\sigma_{w f}$ & $\begin{array}{l}\text { Wrinkling stress in the face material of } \\
\text { sandwich beam }\end{array}$ \\
\hline$\sigma_{y s}$ & Yield strength of foam's cell-wall material \\
\hline$\sigma_{y f}$ & $\begin{array}{l}\text { Yield strength of the face material of sandwich } \\
\text { beam }\end{array}$ \\
\hline$\tau_{c}$ & Shear stress in the core of sandwich beam \\
\hline$\varphi$ & Geometric coefficients of foam cell \\
\hline
\end{tabular}

Corresponding numerical models are also developed based on the maximum principal stress criteria to predict the damage in the PMI foam core. The results obtained from different methods are compared and discussed and the conclusions are made at last.

\section{Theoretical predictions of the failure of sandwich beam with PMI foam core in three point bending}

\subsection{The mechanical behavior of PMI foams under compression}

There are two approaches to model the constitutive behavior of the PMI foam materials. The first approach is continuum modeling, namely critical state theory that has been used in the standard finite element analysis (FEA). The second approach is micromechanical modeling, in which the actual cellular structure is modeled. Gibson and Ashby [23], Chen and Lakes [24] have developed the theory of micro-mechanics of cellular materials, in which the crush behaviors of the foams are dependent on the properties of its parent material. Since the major part of PMI foams is in compression [23], this section discusses the constitutive relation of the ROHACELL WF series PMI foams under compressive load on the basis of the micro-mechanics of cellular materials.

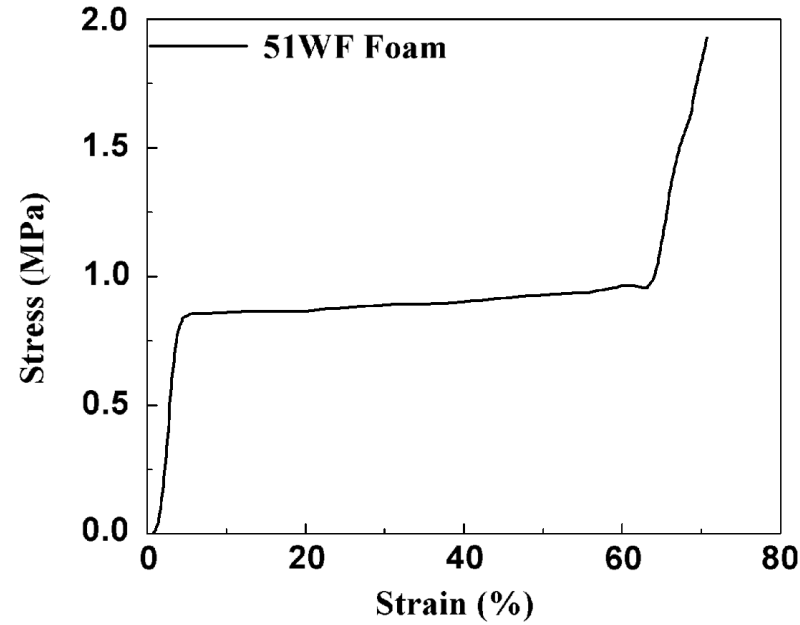

Figure 1. Stress-strain curve of ROHACELL-51WF foam tested under uniaxial compressive load.

Figure 1 shows the typical engineering compressive stressstrain curve of ROHACELL 51WF foam in compression test. The foam material shows elastic behavior under low stress condition followed by a long collapse plateau which is truncated by a high stress densification region. Theory developed by Gibson and Ashby predicts:

$$
\begin{gathered}
\frac{E^{*}}{E_{s}}=C_{1} \phi^{2}\left(\frac{\rho^{*}}{\rho_{s}}\right)^{2}+C_{1}^{\prime}(1-\phi) \frac{\rho^{*}}{\rho_{s}}+\frac{p_{0}\left(1-2 v^{*}\right)}{E_{s}\left(1-\rho^{*} / \rho_{s}\right)} \\
\frac{\sigma_{p l}^{*}}{\sigma_{y s}}=C_{5}\left(\phi \frac{\rho^{*}}{\rho_{s}}\right)^{3 / 2}+C_{5}^{\prime \prime}(1-\phi) \frac{\rho^{*}}{\rho_{s}}+\frac{p_{0}-p_{a t}}{\sigma_{y s}} \\
\varepsilon_{D}=1-1.4 \frac{\rho^{*}}{\rho_{s}}
\end{gathered}
$$

where $E^{*}$ and $E_{s}$ are the Young's modulus of the foam and the cell-wall material, respectively, $\sigma_{p l}^{*}$ and $\sigma_{y s}$ are the compressive crushing strength of the foam and the yield strength of the cellwall material, respectively, $\varepsilon_{D}$ is the critical strain at which the curve's slope becomes $E_{s}, \rho^{*}$ and $\rho_{s}$ are the density of the foam and the cell-wall material, respectively, $p_{0}$ and $p_{a t}$ is the initial fluid pressure and the atmospheric pressure, respectively, $C_{1}$, $C_{1}^{\prime}, \varphi, C_{5}$ and $C_{5}^{\prime \prime}$ are the corresponding coefficients that contain all the geometric constants of the microscopic structure of foam cell [23]. The study of Simone and Gibson [25] shows that for most situations, these parameters can be identified as $C_{1} \approx C_{1}^{\prime} \approx 1, \varphi \approx 0.68, C_{5} \approx 0.3, C_{5}^{\prime \prime} \approx 0.44$. The first and second terms of equations (1.1a) and (1.1b) describe the combined effect of cell-edge bending and cell-face stretching while the third term is caused by the compression of cell fluid. In man-made foams, $p_{0}$ is usually close to $p_{a t}$ that is insignificant compared to $E^{*}$ and $\sigma_{p l}^{*}$, so the third terms' contribution is negligible [23].

In Chen and Lakes's micro-mechanics model [24] for ROHACELL closed-cell foams based on the tetrakaidecahedral 
Table 1. Comparison between micro-mechanics prediction and experimental results.

\begin{tabular}{|c|c|c|c|c|c|c|c|c|c|}
\hline \multirow[t]{2}{*}{ Type } & \multirow{2}{*}{$\begin{array}{l}\text { Density } \\
\left(\mathrm{Kg} / \mathrm{m}^{3}\right)\end{array}$} & \multicolumn{4}{|c|}{$E^{*}(\mathrm{MPa})$} & \multicolumn{4}{|c|}{$\sigma_{p l}^{*}(\mathrm{MPa})$} \\
\hline & & $\begin{array}{c}\text { Equation } \\
\text { (1.1a) }\end{array}$ & $\begin{array}{c}\text { Equation } \\
(1.2 \mathrm{a})\end{array}$ & $\begin{array}{c}\text { Equation } \\
(1.3 \mathrm{a})\end{array}$ & $\begin{array}{c}\text { Test } \\
\text { result }\end{array}$ & $\begin{array}{c}\text { Equation } \\
(1.1 b)\end{array}$ & $\begin{array}{c}\text { Equation } \\
(1.2 b)\end{array}$ & $\begin{array}{c}\text { Equation } \\
(1.3 b)\end{array}$ & $\begin{array}{c}\text { Test } \\
\text { result }\end{array}$ \\
\hline $51 \mathrm{WF}$ & 52 & 76 & 117 & 76 & 75 & 0.69 & 0.82 & 0.79 & 0.8 \\
\hline $71 \mathrm{WF}$ & 75 & 113 & 171 & 113 & 105 & 1.03 & 1.22 & 1.68 & 1.7 \\
\hline $110 \mathrm{WF}$ & 110 & 173 & 257 & 172 & 180 & 1.58 & 1.91 & 3.48 & 3.6 \\
\hline $200 \mathrm{WF}$ & 205 & 354 & 503 & 352 & 350 & 3.23 & 4.10 & 10.42 & 9.0 \\
\hline
\end{tabular}

unit cell shape, equations (1.1a) and (1.1b) can be written as follows:

$$
\begin{gathered}
\frac{E^{*}}{E_{s}}=\frac{0.343+0.823\left(\rho^{*} / \rho_{s}\right)}{0.684+\rho^{*} / \rho_{s}} \times \frac{\rho^{*}}{\rho_{s}} \\
\frac{\sigma_{p l}^{*}}{\sigma_{y s}}=0.454\left(0.417+\frac{\rho^{*}}{\rho_{s}}\right) \frac{\rho^{*}}{\rho_{s}}
\end{gathered}
$$

where $E^{*}, E_{s}, \sigma_{p l}^{*}, \sigma_{y s}, \rho^{*}$ and $\rho_{s}$ represent the same as in equations (1.1a)-(1.1c).

However, data derived from equations (1.1) and (1.2) show some disagreements compared to the test results of the ROHACELL WF series foams. One of the probable reasons is that the unit cell models used in these theories are different from the real situation, which means the geometric coefficients of the microscopic foam cell $C_{1}, C_{1}^{\prime}, \varphi, C_{5}$ and $C_{5}^{\prime \prime}$ shall be reconsidered. Assuming that for each type of ROHACELL WF series foams the foregoing geometric coefficients share the same value since nothing but the foams' porosities changed during the manufacturing processes of foams. Therefore, the Young's moduli of foams and compressive crushing strengths are only dependent on their relative densities. Equations (1.1a) and (1.1b) can be rewritten as:

$$
\begin{aligned}
& \frac{E^{*}}{E_{s}}=A_{1}\left(\frac{\rho^{*}}{\rho_{s}}\right)^{2}+B_{1} \frac{\rho^{*}}{\rho_{s}} \\
& \frac{\sigma_{p l}^{*}}{\sigma_{y s}}=A_{2}\left(\frac{\rho^{*}}{\rho_{s}}\right)^{32}+B_{2} \frac{\rho^{*}}{\rho_{s}}
\end{aligned}
$$

where $A_{1}, B_{1}, A_{2}, B_{2}$ can be determined by two sets of test data. Based on the test results of the ROHACELL 51WF and ROHACELL 71WF PMI foam [26], coefficients $A_{1}, B_{1}, A_{2}$ and $B_{2}$ are $0.44,0.32,2.32$ and -0.28 , respectively. Table 1 shows the Young's modulus $\left(E^{*}\right)$ and the compressive crushing strength $\left(\sigma_{p l}^{*}\right)$ of different types of ROHACELL-WF foams given by equation (1.1)-(1.3)and from tests results under uniaxial compressive load. It can be seen that the test results are in good agreement with the predictions from equation (1.3).

For closed-cell foams the post-collapse plateau rises because the enclosed gas is compressed when the cells collapse, creating a restoring pressure. The post-collapse curve can be described by [23]:

$$
\sigma^{*}=\sigma_{p l}^{*}+\frac{p_{0} \varepsilon}{1-\varepsilon-\rho^{*} / \rho_{s}}
$$

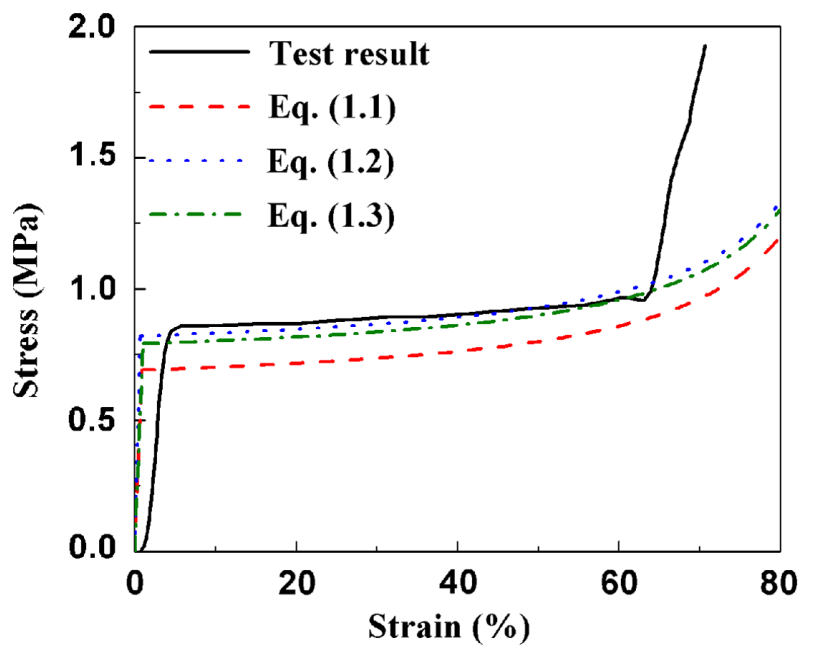

Figure 2. Compress stress-strain curve of ROHACELL-51WF PMI foam.

the second term in the right-hand side of the equation causes the plateau stress to rise with strain. Base on the data derived from equations (1.1)-(1.3) and equation (1.4), the compressive stress-strain curves of ROHACELL 51WF foams can be predicted as shown in Figure 2.

\subsection{Failure mechanism of the sandwich beam with PMI foam core in three point bending}

Basic mechanical properties of sandwich beams have been described in many researches [23, 27, 28]. A sandwich beam model in three-point bending is provided in Figure 3. To analyze the failure mechanism of beams, the normal stress and shear stress (results of specific moment and shear being applied) acting in the face and core needs to be characterized. Considering the shear stress as linear through the faces and constant through the core since the faces are much stiffer and thinner than the core, the normal and shear stresses can be expressed as [28]:

$$
\begin{gathered}
\sigma_{f}=\frac{M E_{f} \times y}{(E I)_{e q}} \\
\sigma_{c}=\frac{M E^{*} \times y}{(E I)_{e q}} \\
\tau_{c}=\frac{Q}{b c}
\end{gathered}
$$




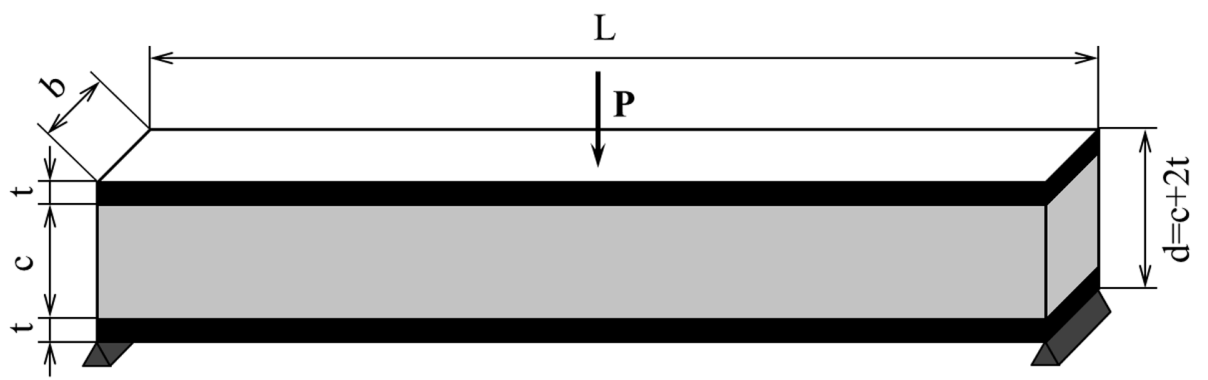

Figure 3. A sandwich beam in three-point bending load.

Table 2. Formulas of three failure modes' critical load.

\begin{tabular}{lcr}
\hline Failure mode & Critical load & Equation number \\
\hline Face yielding & $P=\frac{4 b t c}{l} \times \sigma_{y f}$ & $(2.6 \mathrm{a})$ \\
Face wrinkling & $P=\frac{4 b t c}{l} E_{f}^{1 / 3} E_{s}^{2 / 3}\left[0.28\left(\frac{\rho^{*}}{\rho_{s}}\right)^{4 / 3}+0.20\left(\frac{\rho^{*}}{\rho_{s}}\right)^{2 / 3}\right]$ & $(2.6 \mathrm{~b})$ \\
Core shearing & $P=2 b c\left[2.32\left(\frac{\rho^{*}}{\rho_{s}}\right)^{3 / 2}-0.28 \frac{\rho^{*}}{\rho_{s}}\right] \sigma_{y s}$ & $(2.6 \mathrm{c})$ \\
\hline
\end{tabular}

where $\sigma_{f}$ and $\sigma_{c}$ are the normal stresses in faces and core respectively, $\tau_{c}$ is the shear stress in core; $M$ and $Q$ are the applied moment and shear, $y$ is the distance from the neutral axis. $(E I)_{e q}$ is the equivalent flexural rigidity, which can be expressed as [28]:

$$
(E I)_{e q}=\frac{E_{f} b t^{3}}{6}+\frac{E^{*} b c^{3}}{12}+\frac{E_{f} b t d^{2}}{2} \approx \frac{E_{f} b c^{2}}{2}
$$

The maximum stress occurs in the cross section which has the maximum moment. In the load case of three-point bending, the maximum moment and the shear can be easily acquired in terms of the concentrated load $P$, that

$$
M=\frac{P l}{4}, Q=\frac{P}{2}
$$

Thus considering equations (2.2) and (2.3), the normal stresses in equation (2.1) can be replaced by the following:

$$
\begin{gathered}
\sigma_{f}=\frac{P l}{4 b t c} \\
\sigma_{c}=\frac{P l}{4 b t c} \times \frac{E^{*}}{E_{f}} \\
\tau_{c}=\frac{P}{2 b c}
\end{gathered}
$$

Several failure modes have been identified for sandwich beams in three point bending [23, 27]: (a) plastic yielding of faces, (b) wrinkling of the compressive face, (c) shearing of the core, (d) face debonding, (e) indentation of the loading point. Since the strength of the adhesive (like epoxy resin) is usually higher than the foam core, the mode of face debonding rarely occurs as long as the adhesive is free of defects. While the last mode only occurs when the loading is extremely localized and can be avoided by increasing the loading area. Hence, the failure modes of (d) and (e) will not be discussed in this paper.

For the first failure mode (a), when the maximum normal stress in faces reaches the yield strength of the face material the faces start to yield. In this case:

$$
\sigma_{f}=\sigma_{y f}
$$

where $\sigma_{y f}$ is the yield strength of face material.

For the second failure mode (b), when the maximum normal stress in faces reaches the local elastic instability stress the faces start to wrinkle. In this case:

$$
\sigma_{f}=\sigma_{w f}
$$

where $\sigma_{w f}$ is wrinkling stress in the face material which can be expressed as follow [28]:

$$
\sigma_{w f}=\frac{3 E_{f}^{1 / 3} E^{* 2 / 3}}{\left[12\left(3-v^{*}\right)^{2}\left(1+v^{*}\right)^{2}\right]^{1 / 3}}
$$

For the last failure mode (c), if the shearing stress in the core is larger than the normal stress in faces during the loading process, the core shearing failure will happen when the shearing stress in the core reaches the yield strength of core material:

$$
\tau_{c}=\sigma_{p l}^{*}
$$

where $\sigma_{p l}^{*}$ is the yielding strength of core materials mentioned in equation (1.3b).

Based on equations (2.5), (2.4) and (1.3) and assuming that the Poisson's ratio $v^{*}$ of PMI foam is zero, the critical loads of different failure modes of the sandwich beams with PMI foam core in three point bending tests can be identified, as listed in Table 2. It can be seen that each equation contains two types of variables: one is related the properties 
Table 3. Information on beam's geometry and face material's properties.

\begin{tabular}{|c|c|c|c|c|c|c|c|c|c|c|}
\hline \multirow[t]{2}{*}{ Item } & \multicolumn{3}{|c|}{ Beam's geometry $(\mathrm{mm})$} & \multicolumn{3}{|c|}{ Face material $(\mathrm{MPa})$} & \multicolumn{4}{|c|}{ Core material $\left(\mathrm{kg} / \mathrm{m}^{3}, \mathrm{MPa}\right)$} \\
\hline & $b$ & $c$ & $t$ & $E_{f}$ & $E_{f 3}$ & $\sigma_{y f}$ & $\rho^{*}$ & $\rho_{s}$ & $E_{s}$ & $\sigma_{y s}$ \\
\hline $\begin{array}{l}51 \mathrm{WF} \\
110 \mathrm{WF}\end{array}$ & 60 & 20 & 1.2 & 97.9 & 42.1 & 792 & $\begin{array}{c}0.052 \\
0.11\end{array}$ & 1.2 & 5200 & 90 \\
\hline
\end{tabular}
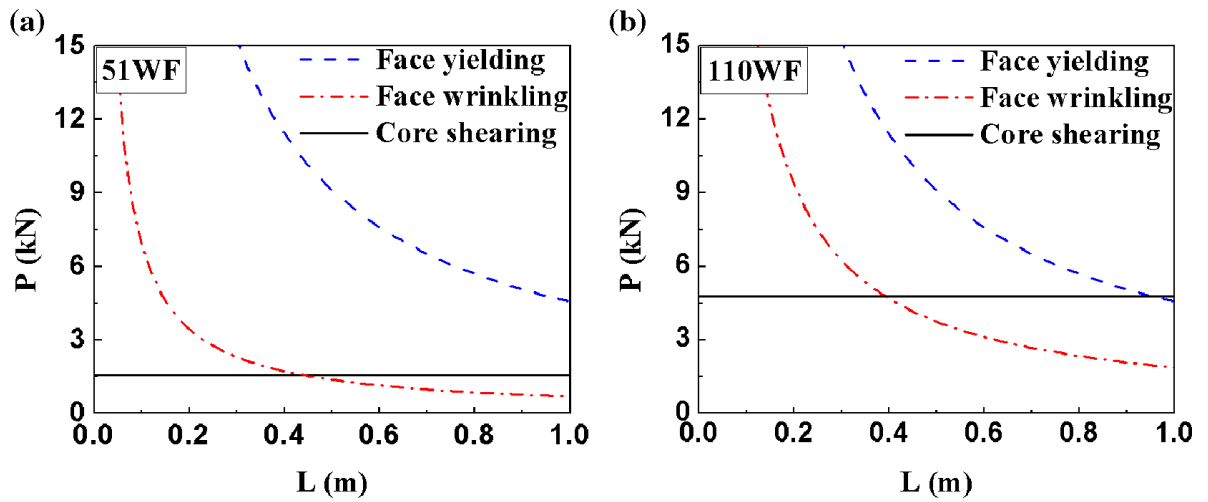

Figure 4. The PMI foam core sandwich beams' failure loads of different failure modes. (a) PMI foam of ROHACELL 51WF; (b) PMI foam ROHACELL 110WF.

of the beam's materials, such as $\sigma_{y s}, \sigma_{y f}, E_{s}$ and $E_{f}$; the other is related the beam's geometrical sizes, including $b, t, c$ and $l$. Besides, from Table 2 it also can be implied that the critical loads of the face yielding and face wrinkling decrease as the span length 1 increase, while the critical load of the core shearing failure remains saturated.

Now take the sandwich beam with PMI foam core and carbon fiber skin sheets as example. Utilizing the formulas from Table 2 the failure load and failure mode of the above sandwich beam can be predicted. Two types of PMI foam are selected as the core material: ROHACELL 51WF and ROHACELL 110WF. According to Table 2, for the face material, the Young's modulus and yielding strength along the beam's extension direction are needed to calculate the critical loads of face yielding and face wrinkling. For the PMI foam core, the foam density as well as the density of parent material, Young's modulus and yielding strength are needed to calculate the critical load of core shearing. Information on beam's geometry and material's properties are listed in Table 3. Figure 4 plots the above PMI foam core sandwich beams' failure loads of different failure modes in terms of the span length.

According to Figure 4, it can be implied that the sandwich beams with both types (51WF and 110WF) of PMI foam cores fail in the mode of core shearing (the black line) under small span situation and in the mode of face wrinkling (the red dash-point curve) under large span situation. The transition span length for the sandwich beam with ROHCELL 51WF foam is $443 \mathrm{~mm}$ and for the sandwich beam with ROHACELL 110WF is $391 \mathrm{~mm}$. In addition, the failure mode of face yielding has less chance to happen in these sandwich beams as the corresponding failure limit is much higher than the other two modes.

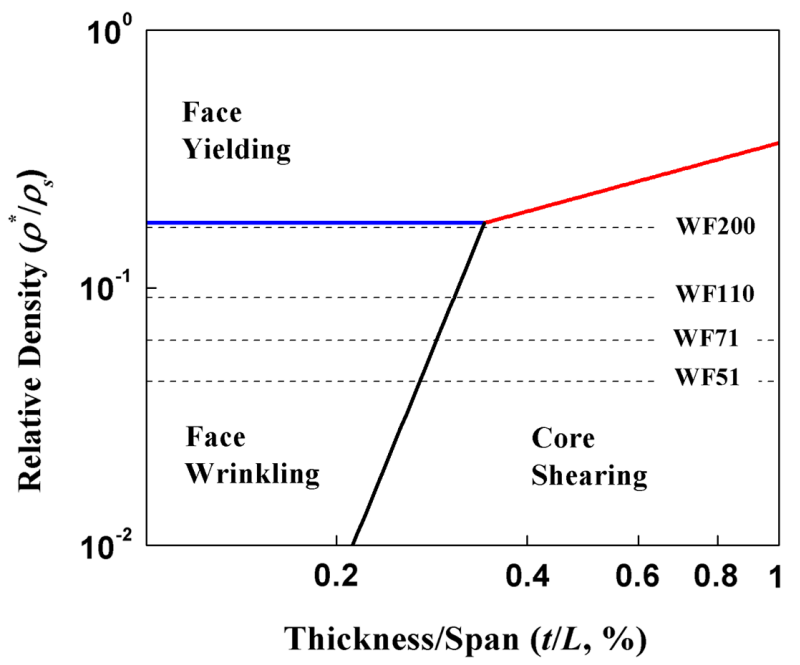

Figure 5. Failure mode map of sandwich beam with PMI foam core and carbon fiber skin sheets.

Given a specific value of the beam's core thickness and width, the failure mode map of the sandwich beam with PMI foam core and carbon fiber skin sheets can be obtained in terms of the relative density of foam and the ratio of beam thickness to span length, as shown in Figure 5. Here the relative density of foam is defined as the ratio of density of foam to density of parent material. From the figure it can be seen that all the ROHACELL WF series foams are out of the face yielding zone, therefore, it can be implied that for any type of PMI foam, the foregoing sandwich beam has only two failure modes, which are face wrinkling and core shearing. 
Table 4. The failure loads of the tested sandwich beams.

\begin{tabular}{lccccc}
\hline $\begin{array}{l}\text { Span } \\
(\mathrm{mm})\end{array}$ & $\begin{array}{c}\text { Specimen } \\
\text { I }\end{array}$ & $\begin{array}{c}\text { Specimen } \\
\text { II }\end{array}$ & $\begin{array}{c}\text { Specimen } \\
\text { III }\end{array}$ & $\begin{array}{c}\text { Specimen } \\
\text { IV }\end{array}$ & Average \\
\hline 140 & 4.48 & 4.47 & 4.92 & 4.19 & 4.52 \\
500 & 3.29 & 3.91 & 3.69 & 3.6 & 3.62 \\
\hline
\end{tabular}

\section{Tests of PMI foam core sandwich beam in three point bending}

The testing sandwich beams consist of ROHACELL 110WF PMI foam core and carbon fiber skin sheets and the core was bonded to the skin layers using a filled epoxy resin. Geometry and materials properties of the specimens can be found in Table 4. The specimens are cut up from a whole sandwich panel and divided into two groups with span lengths of $140 \mathrm{~mm}$ and $500 \mathrm{~mm}$. Each group contains four specimens. All tests were performed on an MTS test machine at a quasi-static loading rate of $2 \mathrm{~mm} / \mathrm{min}$ according to the standard GB/T 1456-2005 of China [29], as shown in Figures 6a and $6 \mathrm{~b}$. The displacement and reacting force at the midpoint can be measured using the transducers set in the loading cell. An Olympus high-speed camera device was set up to capture the failure process in beams, as shown in Figure 6c. The camera's maximal frame rate is 33,000 frames per second. To guarantee the image quality, the shooting frame rate is finally set to 10,000 frames per second so that all details can been caught during the failure process.

The force-displacements curves of the testing sandwich beams with span lengths of $140 \mathrm{~mm}$ and $500 \mathrm{~mm}$ are shown in Figure 7, where a non-linear feature is observed at the beginning of the tests, which is caused by the seating effect on the loading cell and supports. However, the non-linear feature does not affect the measurement of the failure limit of beams and the critical failure load of each testing sandwich is determined by the maximum value of the force-displacements curve, as listed in Table 4.

Figure 8 shows the image of the $140 \mathrm{~mm}$-span length beam tested in three-point bending load until failure. From the image it can be seen that there is a $45^{\circ}$ crack progressed from the loading cell to the bottom face, which is followed by a horizontal crack that ends at the support point. It is clear that the beam fails in the core shearing mode, which is same as the theoretical prediction in the previous section. The failure of material occurs almost instantaneous and the high speed camera was unable to capture the failure process under the setting of 10,000 frames per second, which means that the failure duration is less than $0.1 \mathrm{~ms}$. In addition, the failure only occurs on one side of the beam since the loading situation can hardly be exactly symmetrical.

Figure 9 shows a group of images of failure process in the testing sandwich beams with $500 \mathrm{~mm}$ span length, which are captured by the high speed camera as above-mentioned. The failure occurs on one side of the beam because of the asymmetrical loading situation. The images show that the upper face adjacent to the loading cell wrinkled at the beginning of the failure process and the wrinkle deformation caused the upper face debond from the core and a crack thereby initiated between the upper face and the core. The crack propagates

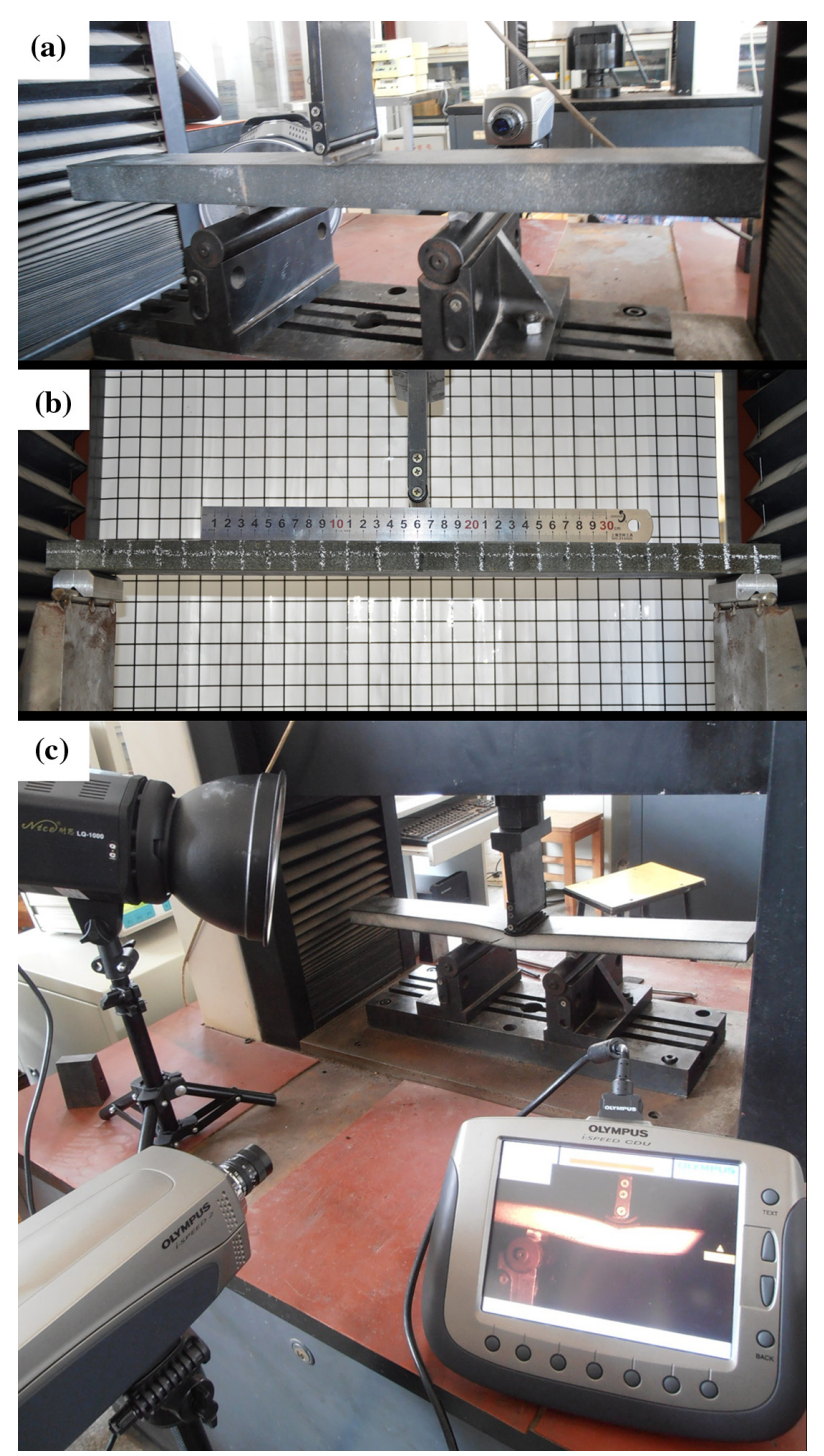

Figure 6. Three-point bending load tests of PMI foam core sandwich beams. (a) $500 \mathrm{~mm}$ span length, (b) $140 \mathrm{~mm}$ span length (c) the testing system and the high speed camera.

along the axial direction towards the support end and large area of the upper face was separated from the core material. Figure 10 gives the failure of the $500 \mathrm{~mm}$ span length sandwich beam. It can be found from Figure 10 that a thin PMI foam layer with numerous surface irregularities adhere to the upper face, which indicates that the adhesive (epoxy resin) between the face and core remain intact and the face debonding failure mode does not occur. Hence, the principle failure mode of sandwich beam with $500 \mathrm{~mm}$ span length is face wrinkling according to the experimental findings.

The sandwich beams consisted of ROHACELL 110WF PMI foam core and carbon fiber skin sheets in three point bending show different failure mode under different span length. The sandwich beam with $140 \mathrm{~mm}$ span length fails in the core shearing mode, while the sandwich beam with $500 \mathrm{~mm}$ span length shows a complex failure process: The face adjacent to the loading cell wrinkled at first and the excessive wrinkling deformation caused damage of the nearby foam 

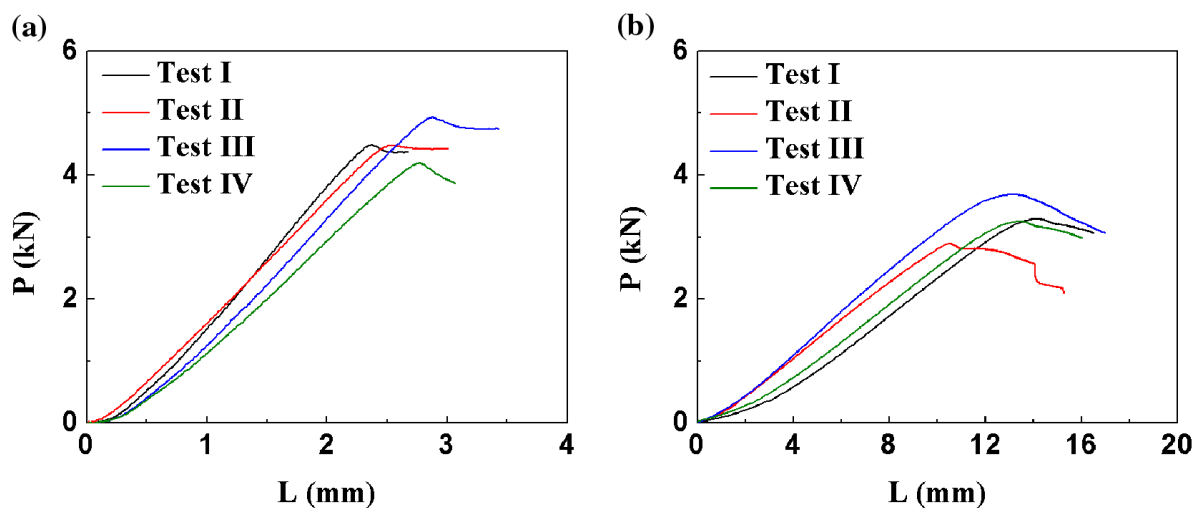

Figure 7. Force-displacement curve of the tested sandwich beams (a) span length of $140 \mathrm{~mm}$ (b) span length of $500 \mathrm{~mm}$.

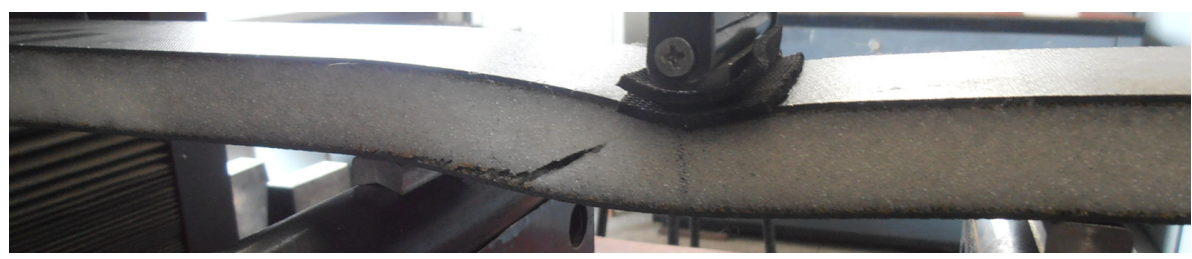

Figure 8. The failure of the sandwich beam of $140 \mathrm{~mm}$ span length.

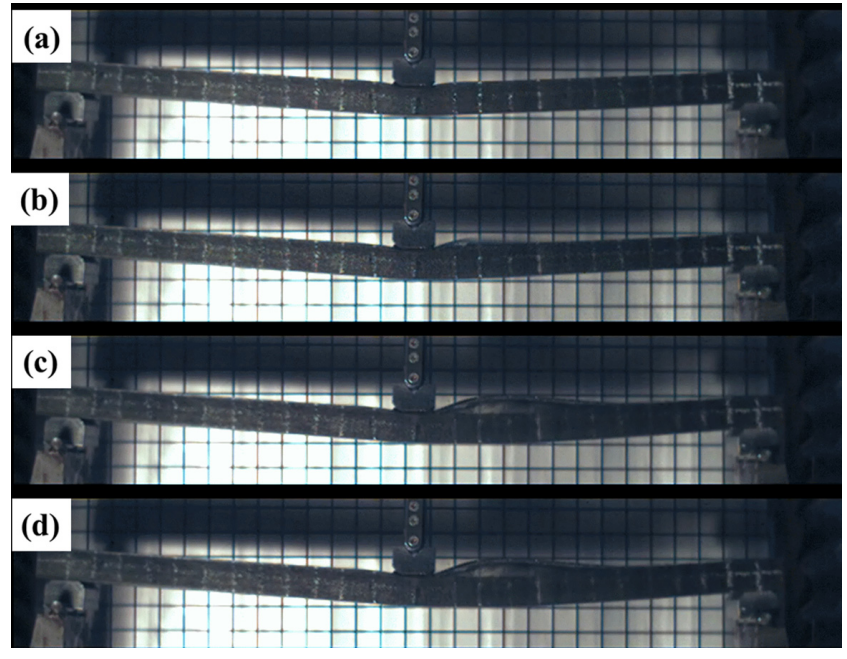

Figure 9. The failure process of the tested sandwich beam with $500 \mathrm{~mm}$ span length captured by high-speed camera.

material until most of the upper face was separated from the core. However, the principle failure mode of sandwich beams with $500 \mathrm{~mm}$ span length is face wrinkling. These above tests results are in agreement with the theoretical predictions that the beam fails in core shearing mode under small span situation, and in face wrinkling mode under large span situation as discussed in Section 1.

\section{Numerical simulations of the sandwich beams with PMI foam core in three point bending}

ABAQUS/Explicit is used here to simulate the failure of the sandwich beam consists of ROHACELL 110WF PMI foam core and carbon fiber skin sheets. The 2-D plain strain models of the sandwich beams with $140 \mathrm{~mm}$ and $500 \mathrm{~mm}$ span lengths are developed. The mechanical behavior of the PMI foam is described by the "low density foam" material model where the maximum principal stress criteria is used to model the failure in the core material while the carbon fiber skin sheets are considered as isotropic materials.

\subsection{Numerical model}

Since the face debond failure is not considered as discussed in Section 1, the adhesive in the beam structure is not included in the model. Thus the core and faces of sandwich beam are modeled as one part, which means the core and faces share



Figure 10. The failure of the sandwich beam of $500 \mathrm{~mm}$ span length. 
(a)



(b)

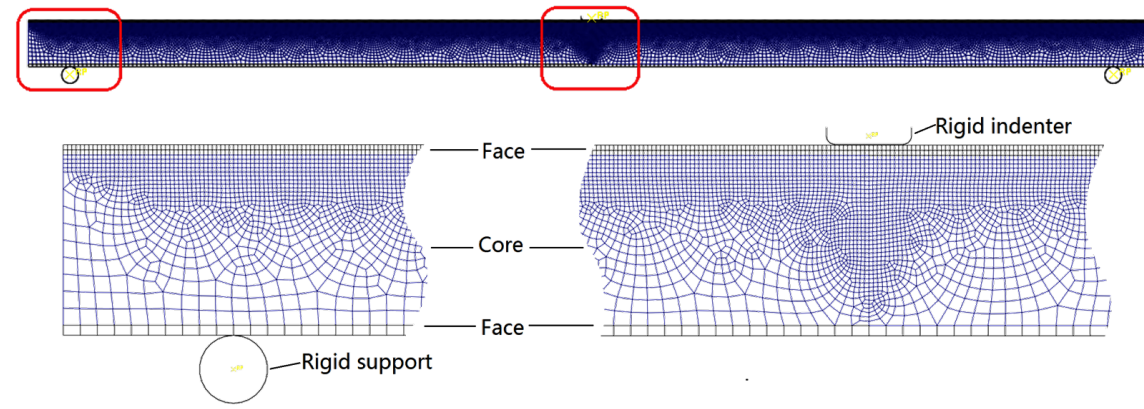

Figure 11. Numerical models of the PMI foam core sandwich beams (a) span length of $140 \mathrm{~mm}$ (b) span length of $500 \mathrm{~mm}$.
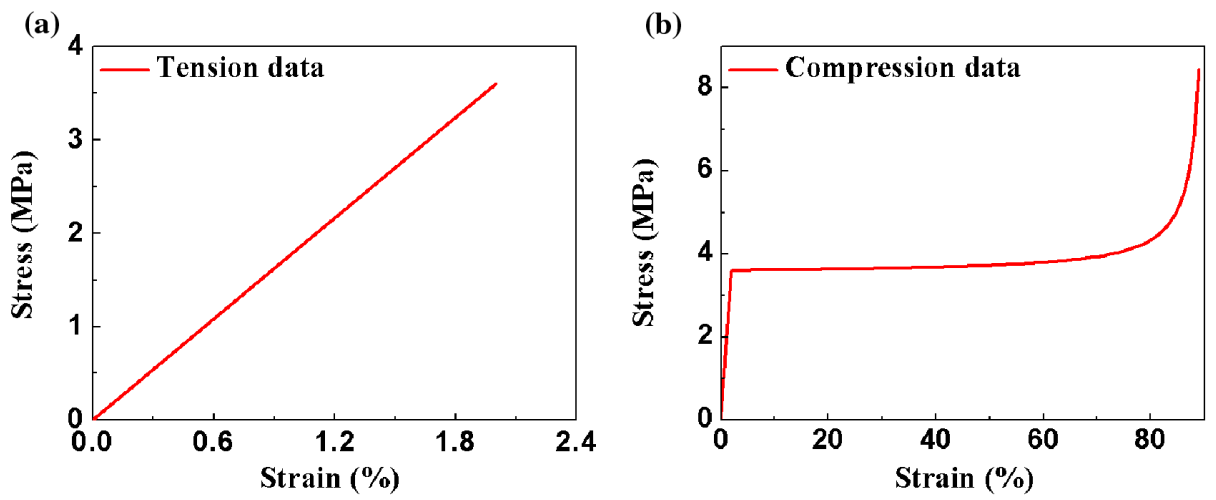

Figure 12. Stress-strain curves of ROHACELL 110WF under uniaxial tension and compression load.
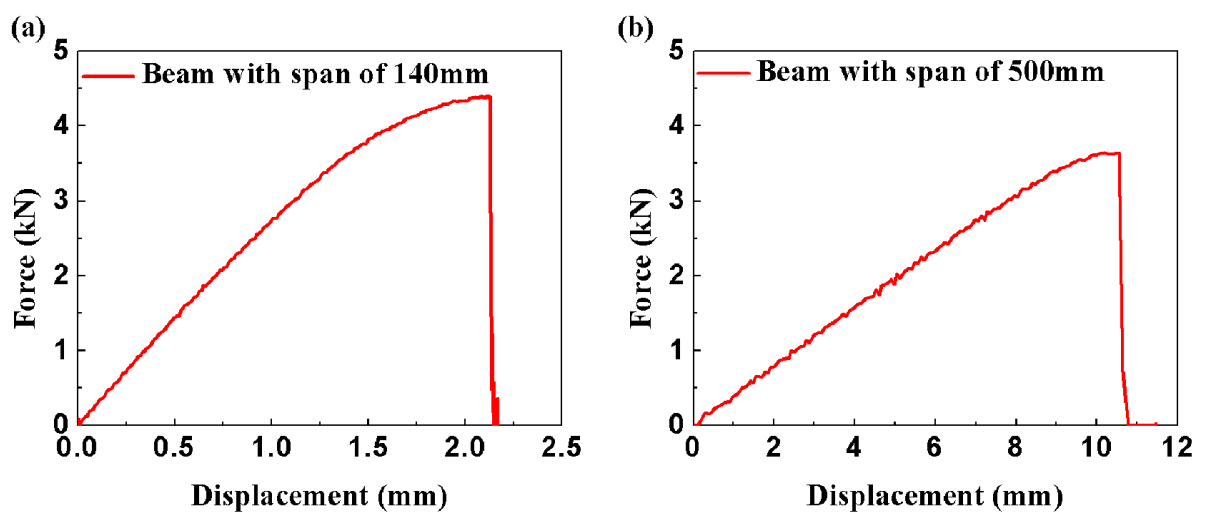

Figure 13. Force-displacement curves of the ROHACELL 110WF core sandwich beams in three point bending (a) span length of 140 mm (b) span length of $500 \mathrm{~mm}$. 
(a)

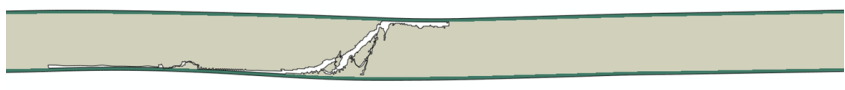

(b)

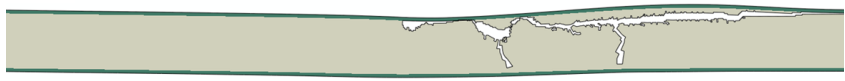

Figure 14. Numerical simulations of the failure the ROHACELL $110 \mathrm{WF}$ core sandwich beams in three point bending (a) span length of $140 \mathrm{~mm}$ (b) span length of $500 \mathrm{~mm}$.

Table 5. Numerical results of the ROHACELL 110WF core sandwich beams in three point bending.

\begin{tabular}{lcl}
\hline Span $(\mathrm{mm})$ & Load capability $(\mathrm{kN})$ & Failure mode \\
\hline 140 & 4.8 & Core shearing \\
500 & 3.6 & Face wrinkling \\
\hline
\end{tabular}

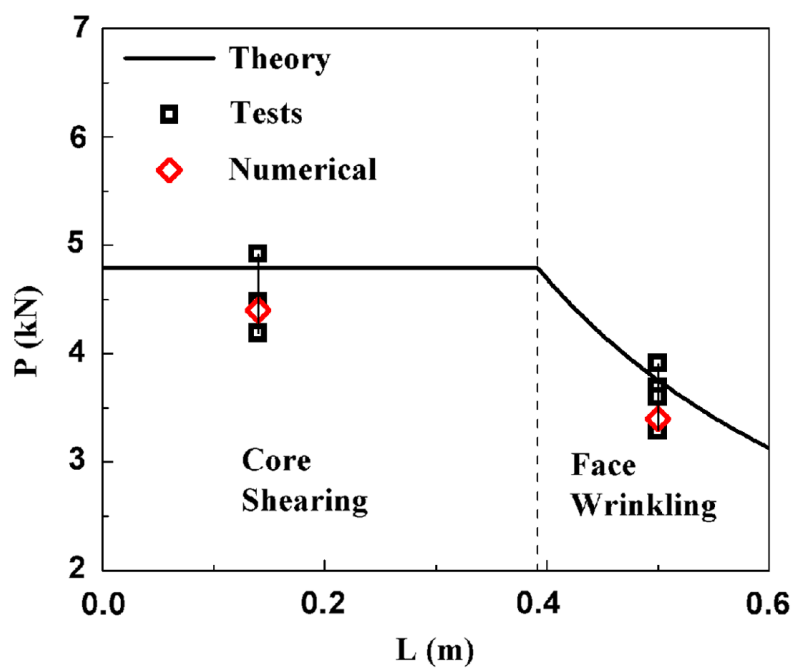

Figure 15. The failure loads of the sandwich beams with ROHACELL 110WF PMI foam core and carbon fiber skin sheets in bending.

the common nodes in the surfaces where they are bonded, as shown in Figure 11. To ensure the precision of simulation, the mesh in the failure area of the beam is refined based on the test results in Section 2. The global mesh size is $1 \mathrm{~mm}$ while the size of the refined mesh is $0.4 \mathrm{~mm}$. The 4-node bilinear plane strain quadrilateral, reduced integration, hourglass control element (CPE4R) is used. The loading cell and supports are regard as rigid while the friction-free contact is set for the interaction between the rigid and the deformable body. All the DOFs of the supports are fixed and the loading cell is applied with a displacement load which is large enough to cause failure of the beam. The nonlinearity geometric analysis (Nlgeom) option is adopted in the whole simulating process.

The mechanical properties of the carbon fiber is described by the orthotropic material with the Young's modulus of the principal direction and thickness direction and the failure stress been given in Table 4. The mechanical property of the PMI foam is described by the Low Density Foam model. The uniaxial tension and compression test data that derived from the theoretical analysis are used as the input of model, as shown in Figure 12. Noticing that the stress-strain curve of PMI foam under uniaxial tension is treated as linear $[3,23]$. The maximum principal stress criteria is used to model the failure in the PMI foam core and the elements that exceed the maximum allowable principal tensile stress, which is $3.7 \mathrm{MPa}$ according to the tests [26], will be removed during the simulation process.

\subsection{Simulation results}

The force-displacement curves of the PMI foam core sandwich beams with span lengths of $140 \mathrm{~mm}$ and $500 \mathrm{~mm}$ are shown in Figure 13. The figure indicates that the load capabilities of the beam of $140 \mathrm{~mm}$ and $500 \mathrm{~mm}$ span length is $5.1 \mathrm{kN}$ and $3.6 \mathrm{kN}$, respectively. The numerical results of PMI foam core sandwich beams tested in three point bending until failure are shown in Figure 14. It can be found that the PMI foam core sandwich beam with span length of $140 \mathrm{~mm}$ fails in core shearing mode, while the sandwich beam with span length of $500 \mathrm{~mm}$ fails in face wrinkling mode (Figure 14).

\section{Results discussion}

The ROHACELL 110WF foam core and carbon fiber skin sheets sandwich beams' load capabilities that derived from theoretical predictions, tests data and numerical simulations are compared in Figure 15, while the beams' failure modes based on test results and numerical simulations are shown in Table 6 .

Table 6. The failure modes of sandwich beams with ROHACELL 110WF PMI foam core and carbon fiber skin sheets in three point bending.

\begin{tabular}{lcc}
\hline Span length & $140 \mathrm{~mm}$ & $500 \mathrm{~mm}$ \\
\hline Numerical & & \\
\cline { 2 - 3 }
\end{tabular}
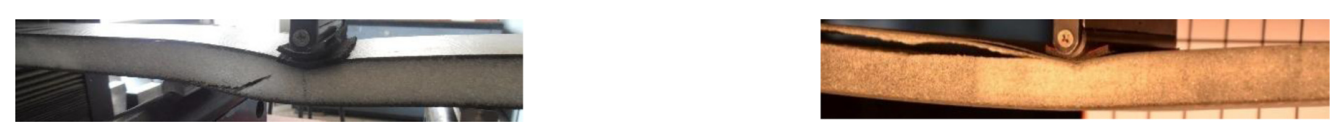


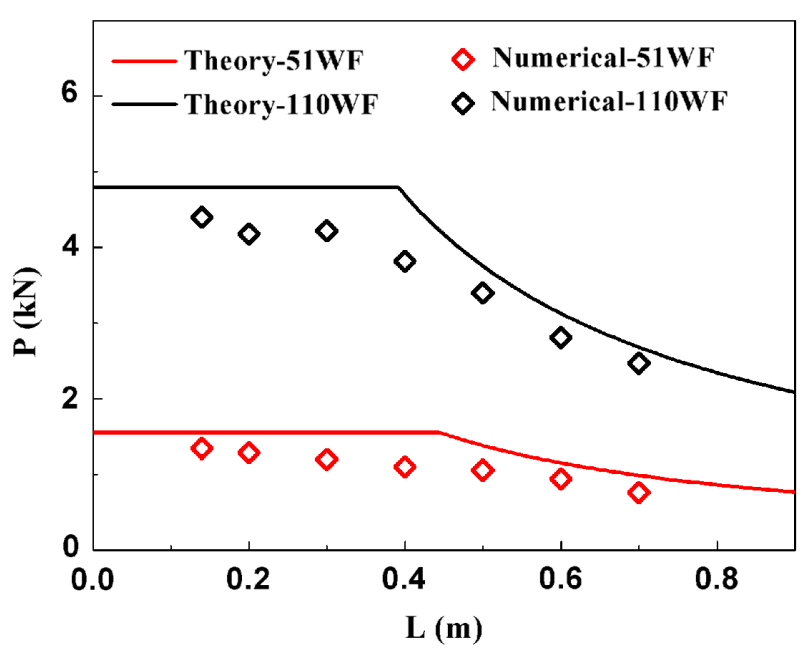

Figure 16. Numerical results of the failure loads of the sandwich beams with different PMI foam cores.

From these figures it can be concluded that the results of different methods show good consistency, which validates the theoretical predictions of the failure of sandwich beams with PMI foam core in three point bending tests. It also proves the feasibility of modeling the failure of beam sandwich structure with PMI foam core based on maximum principal stress criteria in the core material.

The above conclusions give the basis to apply the virtual experiments method to the investigation of PMI foam theory.

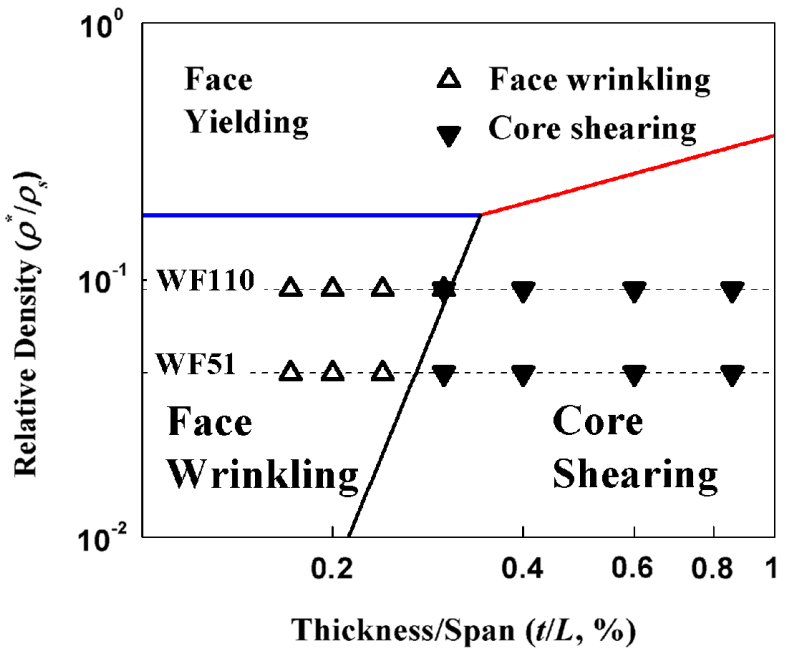

Figure 17. Failure mode map of PMI foam core/carbon-fiber epoxy resin composites sandwich beams.

In order to verify the exactitude of the failure mode map as shown in Figure 5, considerable real tests are needed to provide convincing properties. However, by adopting the above-mentioned hybrid methods (both experimental and numerical), the large amount of tests can be reduced by conducting equivalent numerical simulations where the mechanical properties of the PMI foam are obtained from the theoretical analysis. Herein, the ROHACELL 51WF and ROHACELL 110WF PMI foam are selected as the typical core materials in the

Table 7. Numerical simulations of the failure modes of sandwich beams with different PMI foam cores.

\begin{tabular}{lcc}
\hline Span $(\mathrm{mm})$ & ROHACELL 51WF & ROHACELL 110WF \\
\hline 140 & & \\
\cline { 2 - 3 } & &
\end{tabular}
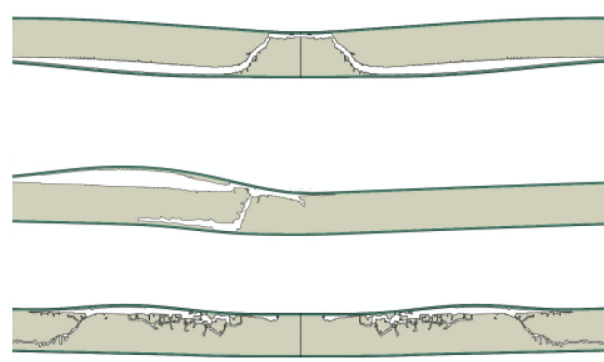
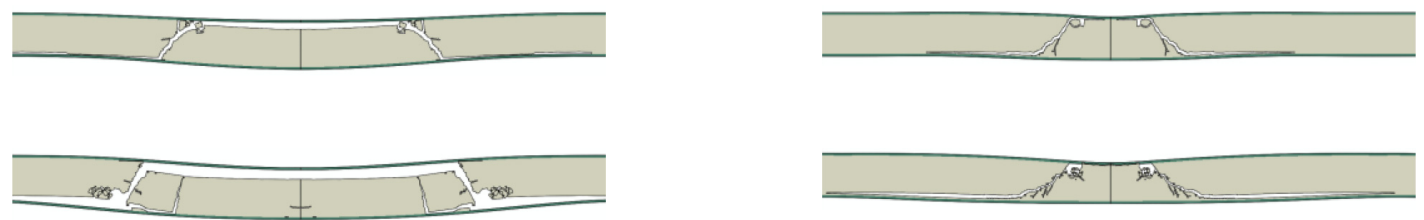
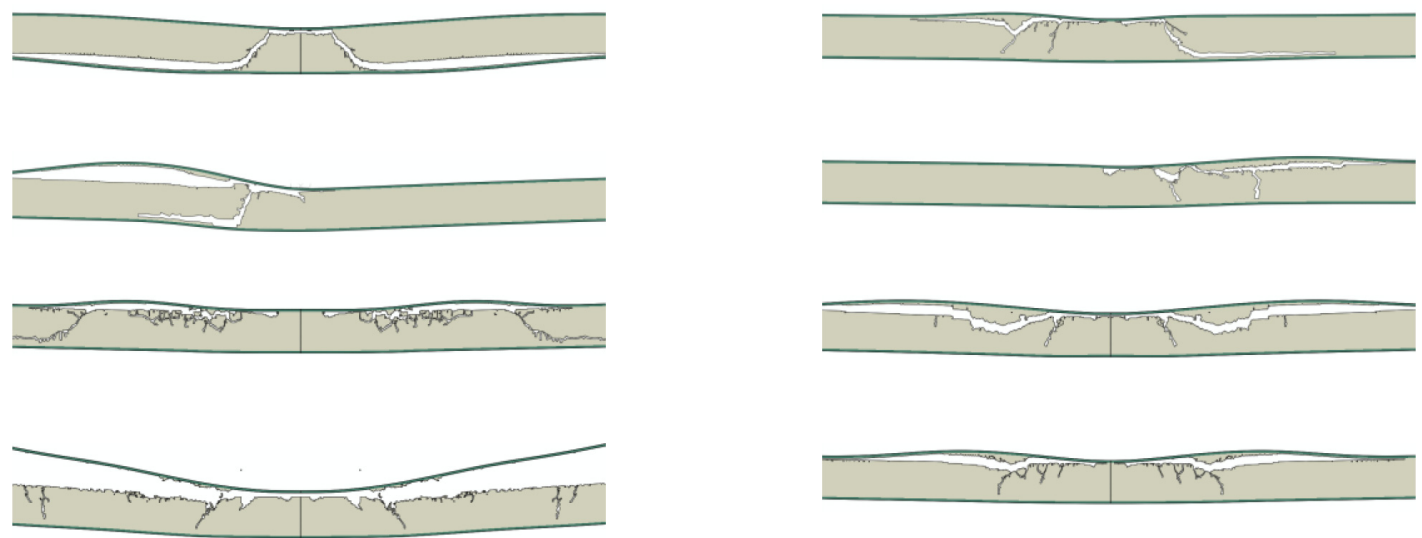
simulations and the skin sheets remain as carbon fiber whose mechanical properties are given in Table 3 , a series of sandwich beams with different span lengths are modeled, in which only half of the beam is modeled while the symmetric boundary condition is applied. The results are given as below.

Figure 16 shows the numerical results of the failure limit of the sandwich beams with different type of PMI foams. The numerical results of failure limit show good consistency with the theoretical predictions. The modes and failure limit of the sandwich beams in bending tests are provided in Table 7. Noticing that for each beam its symmetric model is developed while the result is mirrored about the symmetric plane, except for the sandwich beam with ROHACELL 110WF PMI foam core and span length of $400 \mathrm{~mm}$, whose span is very close to the transition span length of ROHACELL 110WF as mentioned in Section 1. The whole model of the foregoing sandwich beam is developed so that the no details will be missed in its simulation result. It can be seen from Table 7 that for the sandwich beams with ROHACELL 110WF PMI foam core, the principle failure mode is core shearing when the span length is less than $400 \mathrm{~mm}$ and face wrinkling when the span length is larger than $400 \mathrm{~mm}$. When the span length is $400 \mathrm{~mm}$, both of the core shearing and face wrinkling occur to the beam as the critical loads of the two failure modes are very close. As for the sandwich beams with ROHACELL 51WF PMI foam core, core shearing is the principle failure mode when the span length is less than $500 \mathrm{~mm}$. When the span length is greater than or equal to $500 \mathrm{~mm}$, the failure mode becomes face wrinkling. Put the numerical results of the sandwich beam with different PMI foam core into the corresponding positions in the failure mode map as shown in Figure 17, where the simulated failure modes of different sandwich beams agree well with the theoretical predictions.

\section{Conclusions}

The constitutive relation of the PMI foam under compression is studied on the basis of the cellular solids theory [23]. The obtained constitutive relation is then applied to investigate the failure mechanism of sandwich beams with PMI foam core in three point bending tests. The tests of the sandwich beams with ROHACELL 110WF foam core and carbon fiber skin sheets in three point bending tests are carried out and the corresponding numerical models are developed. Theoretical analysis shows that the PMI foam core sandwich beams fail in the mode of core shearing in small span situation while face wrinkling turns out to be the principle failure mode in large span situation, which is validated by the both experimental findings and the numerical modeling. In addition, the images captured by high speed camera show that the failure process of sandwich beams (PMI foam core) with span length of $500 \mathrm{~mm}$ is complex: The face adjacent to the loading cell wrinkles at first and the excessive wrinkling deformation causes the progressive failure of the nearby foam material until most of the upper face was separated from the core material. The present studies provide a theoretic tool for the design of sandwich structures with PMI foam core and give the basis to reduce the requirements of tests by numerical modeling for the validation of design of PMI foam core embedded sandwich beams.

\section{Implications and influences}

The studies provide a theoretic tool for the design of sandwich structures with PMI foam core and give the basis to reduce the requirements of tests by numerical modeling for the validation of design of PMI foam core embedded sandwich beams.

Acknowledgements. The research is supported by the 973 Program (No. 2014CB049000), National Natural Science Foundation of China (11372062), China Postdoctoral Science Foundation (2014M551070), 111 Project (B14013).

\section{References}

1. Seibert HF. 2006. Applications for PMI foams in aerospace sandwich structures. Reinforced Plastics, 50(1), 44-48.

2. Li Q, Mines R. 2002. Strain measures for rigid crushable foam in uniaxial compression. Strain, 38(4), 132-140.

3. Li Q, Mines R, Birch R. 2000. The crush behaviour of Rohacell-51WF structural foam. International Journal of Solids and Structures, 37(43), 6321-6341.

4. Chen C, Anderson W, Lakes R. 1994. Relating the properties of foam to the properties of the solid from which it is made. Cellular Polymers, 13(1), 16-32.

5. Burman M, Zenkert D. 1997. Fatigue of foam core sandwich beams -1 : undamaged specimens. International Journal of Fatigue, 19(7), 551-561.

6. Zenkert D, Burman M. 2009. Tension, compression and shear fatigue of a closed cell polymer foam. Composites Science and Technology, 69(6), 785-792.

7. Zenkert D, Burman M. 2011. Failure mode shifts during constant amplitude fatigue loading of GFRP/foam core sandwich beams. International Journal of Fatigue, 33(2), 217-222.

8. Akay M, Hanna R. 1990. A comparison of honeycomb-core and foam-core carbon-fibre/epoxy sandwich panels. Composites, 21(4), 325-331.

9. Tsang P, Dugundji J. 1992. Damage resistance of graphite/ epoxy sandwich panels under low speed impacts. Journal of the American Helicopter Society, 37(1), 75-81.

10. Bernard ML, Lagace PA. 1989. Impact resistance of composite sandwich plates. Journal of Reinforced Plastics and Composites, 8(5), 432-445.

11. Wu C, Sun C. 1996. Low velocity impact damage in composite sandwich beams. Composite Structures, 34(1), 21-27.

12. Benderly D, Putter S. 2004. Characterization of the shear/ compression failure envelope of Rohacell foam. Polymer Testing, 23(1), 51-57.

13. Kazemahvazi S, Tanner D, Zenkert D. 2009. Corrugated allcomposite sandwich structures. Part 2: Failure mechanisms and experimental programme. Composites Science and Technology, 69(7), 920-925.

14. McGarva L, Åström B. 1999. Experimental investigation of compression moulding of glass/PA12-PMI foam core sandwich components. Composites Part A: Applied Science and Manufacturing, 30(10), 1171-1185. 
15. Can W, Hao-ran C, Zhen-kun L. 2010. Experimental investigation of interfacial fracture behavior in foam core sandwich beams with visco-elastic adhesive interface. Composite Structures, 92(5), 1085-1091.

16. Mamalis A, Spentzas K, Manolakos D, Ioannidis M, Papapostolou D. 2008. Experimental investigation of the collapse modes and the main crushing characteristics of composite sandwich panels subjected to flexural loading. International Journal of Crashworthiness, 13(4), 349-362.

17. Flores-Johnson E, Li Q. 2011. Experimental study of the indentation of sandwich panels with carbon fibre-reinforced polymer face sheets and polymeric foam core. Composites Part B: Engineering, 42(5), 1212-1219.

18. Yang F, Lin Q, Jiang J. 2015. Experimental study on fatigue failure and damage of sandwich structure with PMI foam core. Fatigue \& Fracture of Engineering Materials \& Structures, 38(4), 456-465.

19. Rinker M, John M, Zahlen PC, Schäuble R. 2011. Face sheet debonding in CFRP/PMI sandwich structures under quasi-static and fatigue loading considering residual thermal stress. Engineering Fracture Mechanics, 78(17), 2835-2847.

20. Shipsha A, Hallström S, Zenkert D. 2003. Failure mechanisms and modelling of impact damage in sandwich beams-a 2D approach: part I-experimental investigation. Journal of Sandwich Structures and Materials, 5(1), 7-31.
21. Rizov V, Shipsha A, Zenkert D. 2005. Indentation study of foam core sandwich composite panels. Composite Structures, 69(1), 95-102.

22. Mamalis A, Manolakos D, Ioannidis M, Papapostolou D. 2005. On the crushing response of composite sandwich panels subjected to edgewise compression: experimental. Composite Structures, 71(2), 246-257.

23. Gibson LJ, Ashby MF. 1997. Cellular solids: structure and properties. Cambridge University Press.

24. Chen C, Lakes R. 1995. Analysis of the structure-property relations of foam materials. Cellular Polymers, 14(3), 186-202.

25. Simone A, Gibson L. 1998. Effects of solid distribution on the stiffness and strength of metallic foams. Acta Materialia, 46(6), 2139-2150.

26. Pei H. 2005. ROHACELL Technical Manual. Yingchuang Degussa (China) Investment Co., Ltd. Shanghai Branch: China.

27. Zenkert D. 1997. The handbook of sandwich construction. UK Engineering Materials Advisory Services Ltd/Chameleon Press Ltd: London.

28. Allen G. 1969. Analysis and Design of Structural Sandwich Panels. Pergamon Press: Oxford. p. 1969.

29. GB/T 1456-2005. 2005. Test method for flexural properties of sandwich construction. Standardization Administration of the People's Republic of China: Beijing, China.

Cite this article as: Wang B, Shi Y, Zhou C \& Li T: Failure mechanism of PMI foam core sandwich beam in bending. Int. J. Simul. Multisci. Des. Optim., 2015, 6, A8. 